\title{
Radiatively inefficient accretion in short-period black hole low mass X-ray binaries
}

\author{
Riley M. T. Connors ${ }^{1,2}$ \\ ${ }^{1}$ Department of Physics \& Astronomy, University of Leicester, \\ Leicester, LE1 7RH, UK \\ ${ }^{2}$ Astronomical Institute "Anton Pannekoek", University of Amsterdam, \\ P. O. Box 94249, 1090 GE Amsterdam, The Netherlands
}

email: r.m.t.connors@uva.nl

\begin{abstract}
There is statistical evidence for a dearth of short-period $\left(P_{\text {orb }}<4 \mathrm{~h}\right)$ black hole $(\mathrm{BH})$ low mass X-ray binaries (LMXBs) in the Galaxy. At short periods accretion onto the central object (be it a $\mathrm{BH}$ ) may become inefficient because the cooling timescale of the gas is greater than the accretion timescale (this is the well known ADAF model). The nature of the switch is important in terms of the outburst timescales of transient sources. The switch may be sharp or occur smoothly over time. I show that the dearth can be explained if the switch to inefficiency occurs sharply at some fraction of the Eddington luminosity of the $\mathrm{BH}\left(f L_{E d d}\right)$.
\end{abstract}

\section{Introduction}

Low mass X-ray binaries (LMXBs) are binary stars consisting of a central compact object (a neutron star, NS, or black hole, $\mathrm{BH}$ ) and a low mass secondary star. Due to the strong tidal forces exerted by the central compact object, matter is pulled from the star and forms an accretion disk around the central object. This matter falls toward the central object due to a loss of angular momentum through viscous interactions. These binaries emit X-ray radiation in a regular outburst cycle (on timescales of 10 - 100 days) as matter built up in the disk accretes onto the central object (see King \& Ritter (1998) for details).

The Ritter-Kolb catalog (Ritter \& Kolb 2003) contains data on all the observed LMXBs within the Galaxy. I extract data from this catalog, focusing on the orbital periods of all the systems, and divide it into three samples; (i) all BH LMXBs, (ii) all NS LMXBs, and (iii) transient NS LMXBs only. I plot the cumulative orbital periods distribution (Figure 1) of all three samples and perform K-S statistics. The results show evidence for a dearth of $\mathrm{BH}$ systems at short orbital periods $\left(P_{\text {orb }}<4 \mathrm{~h}\right)$.

\section{Radiatively inefficient accretion: a possible explanation}

I explore a possible explanation for the dearth of short-period BH LMXBs in the Galaxy in the form of a regime of inefficient accretion at sub-Eddingtion luminosities. A well explored model for inefficiency is the advection dominated accretion flow (ADAF) (Narayan \& Yi 1995). This accretion consists of hot low-density gas, where the cooling time exceeds the accretion time, such that the gas falls into the event horizon of the $\mathrm{BH}$ before its energy (built up by viscous heating) can be radiated away. The LMXB is predicted to enter this regime when it's luminosity drops below $\sim 1-10 \%$ of the Eddington luminosity. 


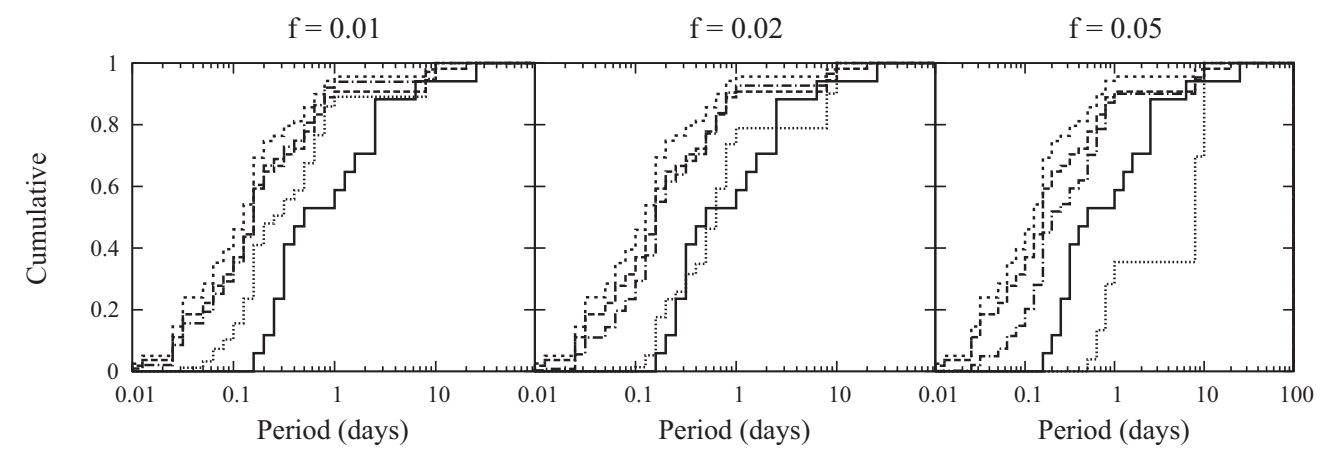

Figure 1. Orbital period distribution of LMXBs in the Galaxy. Plotted are the NS LMXBs from sample 2 (dashed), BH LMXBs from sample 1 (solid line), predicted BH LMXBs with no switch (thick dotted), predicted LMXBs with a sharp (A) switch (thin dotted), and predicted BH LMXBs with a smooth (B) switch (dot-dashed). The three panels show the switches occurring at three different fractions of $L_{\mathrm{Edd}}$.

I consider two cases for a switch to inefficiency; (A) a sharp switch at a fraction of the Eddington luminosity $\left(f L_{E d d}\right)$, and (B) a smooth efficiency decrease at $f L_{E d d}$. In each case since the accretion rate depends on disk radius, and thus the orbital period $\left(R_{D} \propto P_{\text {orb }}^{2 / 3}\right)$, at short periods the peak luminosity of LMXBs drops below the threshold for inefficiency. In this limit BH systems have lower peak luminosities, outburst durations, and duty cycles, and so their detection probabilities are reduced. I use Monte Carlo techniques to simulate the accretion evolution of LMXBs in the Galactic plane (using the prescription of King \& Ritter (1998), and the two cases for a switch to inefficiency), and calculate their detection probabilities as a function of orbital period in order to produce predicted orbital period distributions.

The overall results are shown in Figure 1. The plots show the cumulative period distributions of the BH and NS LMXB samples described above, and the predicted distributions of $\mathrm{BH}$ systems with no switch to inefficiency, a sharp switch to inefficiency (A), and a smooth switch to inefficiency (B). The best fit to the data is achieved for $\mathrm{BH}$ systems with a sharp switch to inefficiency at $0.02 L_{E d d}$ (Figure 1), and thus this switch best explains the Galactic distribution. This can be interpreted as a true decrease in the system's bolometric luminosity, or a decrease in X-ray luminosity (a spectral shift in emission). More improved models and a further analysis can be found in Knevitt et al. (2013). A model that focuses on the Galactic center using empirically determined period distributions could be used to explain the observed Luminosity Function (LF) of LMXBs in this region (see e.g. Fabbiano 2010). Applying theoretical models for accretion to these kinds of population studies could be a useful test of the theories themselves.

\section{Acknowledgements}

The author thanks Gillian Knevitt and Graham Wynn for assistance with this work.

\section{References}

Fabbiano, G. 2010, AIP-CP 1314, 318-325

King, A. R. \& Ritter, H. 1998, MNRAS 293, 42-48

Knevitt, G., Wynn, G. A., Vaughan, S., \& Watson, M. G. 2013, MNRAS, accepted

Narayan, R. \& Yi, I. 1995, ApJ 452, 710

Ritter, H. \& Kolb, U. 2003, A\&A 404, 301 